\title{
Características clínicas, recursos utilizados y costos de la psoriasis en pacientes de una IPS de referencia del Valle de Aburrá, 2014
}

Clinical characteristics, utilization of resources and costs of patients with psoriasis in a medical center referenced in el Valle de Aburrá, 2014

\author{
Uriel Palacios-Barahona', Sol Beatriz Jiménez², John Jairo Orozco \\ 1. Médico investigador, Universidad CES, Centro de Evaluación de Tecnologías en Salud, Medellín, Colombia. \\ 2. Médica dermatóloga; coordinadora, Programa de Dermatología, Universidad CES, Medellín, Colombia.
}

\section{RESUMEN}

Introducción. La caracterización de los pacientes con psoriasis es importante porque permite conocer las formas clínicas más frecuentes y la gravedad de la enfermedad, y además, determinar su impacto en los costos para el sector salud.

objetivo. Determinar las características clínicas, los recursos utilizados y los costos de los pacientes con psoriasis atendidos en un centro de referencia del área metropolitana del Valle de Aburrá en el 2014.

Metodología. Se llevó a cabo un estudio descriptivo de pacientes con psoriasis que asistieron al Centro Dermatológico CES, Sabaneta, entre el $1^{\circ}$ de enero y el 30 de noviembre de 2014. Los recursos utilizados, como citas, tratamientos, exámenes paraclínicos y procedimientos, se consultaron en las historias clínicas. Para calcular las tarifas de consultas y procedimientos, se usaron las establecidas en el manual tarifario ISS 2001 más el 30 \% y en el Seguro Obligatorio de Accidentes de Tránsito (SOAT) 2014; en medicamentos, se utilizó el sistema de información de precios de medicamentos (SISMED).

Resultados. Se incluyeron 95 pacientes, cuya media de edad fue de 47,2 años (desviación estándar, $\mathrm{DE}=15,5$ años); el 47 eran mujeres. El promedio del índice DLQI (Dermatology Life Quality Index) fue de 8,2 (DE=7,2) y el del PASI (Psoriasis Area and Severity Index) fue de 5,7 (DE=4,7).

Los costos mínimo y máximo, según el manual ISS + 30 \%, fueron de COP\$ 21.710 y COP\$ 13’803.505 para la psoriasis leve, y de COP\$ 402.610 y COP\$ 36’643.757 para la moderada a grave; cuando se utilizó terapia biológica, fueron de COP\$ 15’608.272 y COP\$ 36’643.757. Según el manual SOAT, los costos oscilaron entre COP\$ 536.987 y COP\$ 39'312.273 en casos de psoriasis entre moderada a grave y, entre COP\$17’286.259 y COP\$39'312.272, cuando se utilizó terapia biológica.

Conclusión. La psoriasis es una enfermedad que acarrea costos elevados de tratamiento en relación con su gravedad y la eficacia del tratamiento.

PALABRAS CLAVE: psoriasis, costos y análisis de costos, terapia biológica, terapia combinada, estudio observacional, costos de la atención en salud, costo de enfermedad.
Correspondencia:

Uriel Palacios-Barahona

Email:

apalacios@ces.edu.co

Recibido: 17 de abril de 2016

Aceptado: 4 de julio de 2016

Conflictos de intereses.

Esta investigación fue desarrollada por el Centro de Evaluación de Tecnologías en Salud de la Universidad CES y el Centro Dermatológico CES Sabaneta, y fue financiado por Novartis de Colombia S.A.

Los investigadores declaran su total independencia del patrocinador y son los únicos responsables de los métodos, conceptos y resultados presentados en este estudio. Novartis no tuvo ningún papel en el desarrollo de esta investigación. Sol Beatriz Jiménez ha sido conferencista para los laboratorios Pfizer, Novartis, AbbVie y Janssen, y labora en la Unidad de Dermatología de la Universidad CES, donde funciona la Unidad de Fototerapia, pero no recibe honorarios adicionales ni dividendos por esta actividad. 


\section{SUMMARY}

Introduction: The characterization of patients with psoriasis is important because it allows an approach to the most frequent clinical forms and the severity of the illness; besides, it enables the possibility of determining the impact of said illness in terms of cost towards the health system.

Objective: To determine the clinical characteristics, supplies needed and cost of patients with psoriasis that received medical attention in a referral center of the metropolitan area in Valle de Aburrá.

Methodology: A descriptive study in patients with psoriasis that attended the Centro Dermatológico CES, Sabaneta, between the January $1^{\text {st }}$ and November $30^{\text {th }}$, 2014, was carried out. The clinical history was used to consult the utilization of appointments, treatments, lab tests, and procedures; the ISS 2001 rates manual plus $30 \%$ and the SOAT 2014 rates manual were used. To estimate the price of drugs a drug information system (SISMED) was used.

Results: Ninety five patients were analyzed. The average age was 47.2 years $(\mathrm{SD}=15.5) ; 47$ of the patients analyzed were women. The average DLQI was 8.2 $(\mathrm{SD}=7.2)$, and the PASI was $5.7(\mathrm{SD}=4.7)$. The manual cost ISS $+30 \%$ for mild psoriasis presented a minimum value of COP\$ 21,710 and a maximum value of COP\$ 13,803,505; moderate to severe psoriasis was between COP\$ 402,610 and COP\$ $36,643,757$; for patients using biological therapy was between COP\$ 15,608,272 and COP\$ $36,643,757$. According to the SOAT manual, the cost of moderate to severe psoriasis was between COP\$ 536,987 and COP\$ 39,312,273 and for those who used biological therapy was between COP\$ 17,286,259 and COP\$ 39,312,272.

Conclusion: Psoriasis is an illness that represents an elevated cost in treatment in relation to the severity and efficiency of the treatment received.

KEY WORDS: Psoriasis, costs and cost analysis, biological therapy, combined modality therapy, observational study, health care costs, cost of illness.

\section{INTRODUCCIÓN}

La psoriasis se caracteriza por hiperproliferación y diferenciación incompleta de la capa epidérmica ${ }^{1-3}$. Afecta a personas de todas las edades, principalmente adultos, sin diferencias según el sexo, con dos picos de incidencia: el primero, alrededor de los 20 años, y el segundo, entre los 50 y los 60 años ${ }^{1}$. Las manifestaciones clínicas de la enfermedad dificultan las actividades básicas de los pacientes y disminuyen su calidad de vida, lo cual se refleja en baja autoestima, sensación de rechazo y preocupaciones psicosexuales ${ }^{4,5}$.

Los aspectos económicos de la salud son cada vez más importantes en todos los sistemas de salud. Se necesita información sobre los costos para las decisiones terapéuticas y la implementación de políticas de salud. Las evaluaciones son necesarias, con el fin de usar adecuadamente las diferentes opciones de tratamiento. Los estudios de costos de una enfermedad son una herramienta reconocida para determinar la carga de las enfermedades en un país e identificar subgrupos para los cuales las tarifas son particularmente altas, independientemente del sistema de salud. Aunque son pocos los estudios sobre costos de la psoriasis, la información disponible indica considerables consecuencias económicas para el sistema de salud ${ }^{6-10}$.

El Centro Dermatológico CES, Sabaneta, cuenta con más de 15 años de experiencia en el manejo de los pacientes con psoriasis y es un centro ambulatorio de referencia en el manejo de enfermedades dermatológicas en el área metropolitana del Valle de Aburrá. Los pacientes son remitidos con diagnóstico ya definido de psoriasis para su manejo especializado o algunos consultan por primera vez allí. Según la gravedad de la enfermedad, reciben tratamiento tópico, fototerapia, medicamentos sistémicos y productos biológicos, si es necesario. Si no están contraindicados y según la necesidad, se administra fototerapia $\mathrm{u}$ otros medicamentos 
tópicos y sistémicos, y se sigue la guía de manejo de la psoriasis de la Sociedad Colombiana de Dermatología ${ }^{11}$.

\section{MATERIALES Y MÉTODOS}

Se hizo un estudio de tipo descriptivo, en el cual se tomó la información de los pacientes con diagnóstico de psoriasis, atendidos en el Centro Dermatológico del CES, Sabaneta, entre el $1^{\circ}$ de enero y el 30 de noviembre de 2014. Se incluyeron todos aquellos pacientes mayores de 18 años con psoriasis en cualquiera de sus formas clínicas, que consultaron durante este tiempo al centro y que aceptaron voluntariamente la participación en el estudio.

Se incluyeron variables como sexo, edad, estado civil, escolaridad, tiempo de evolución de la enfermedad, tipo de tratamiento recibido, calidad de vida valorada con el DLQI (Dermatology Life Quality Index), puntaje del PASI (Psoriasis Area and Severity Index), superficie de área corporal comprometida, enfermedades asociadas, consultas médicas ambulatorias, procedimientos, exámenes paraclínicos, uso de medicamentos sistémicos y productos biológicos, y costos de consultas médicas ambulatorias, procedimientos, exámenes paraclínicos, medicamentos sistémicos y productos biológicos.

Se elaboró un formato en el que los especialistas recopilaron información sociodemográfica y clínica de interés, extraída de la historia clínica. La información fue digitada en una base de datos de Excel.

Los datos correspondientes a los recursos utilizados fueron consultados por un médico en la historia clínica, quien procedió a contar el número de citas durante el periodo de estudio, así como a identificar el tratamiento recibido para la psoriasis y los exámenes paraclínicos utilizados. Para evitar el sesgo de información, la información faltante fue consultada con el médico tratante para evitar la pérdida de los datos. El trabajo fue aprobado por el Comité de Ética e Investigación de la Universidad CES, siguiendo los enunciados de la declaración de Helsinki.

\section{COSTO DE MEDICAMENTOS}

Para el cálculo de los costos se creó un "caso base”, que equivale al tratamiento de un paciente estándar con psoriasis durante 24 semanas, en su fase inicial de tratamiento de inducción de 16 semanas y la de mantenimiento hasta la semana 24; se hizo un ajuste según la efectividad del tratamiento y el área de superficie corporal comprometida ${ }^{11,12}$. Para calcular el costo de los medicamentos, se siguió la metodología propuesta por el Instituto de Evaluación de Tecnologías en Salud (IETS), consistente en:

a. Identificar el principio activo del medicamento de interés dentro del listado de las circulares emitidas por el Ministerio de Salud.

b. Estimar el precio de la unidad mínima en miligramos o en unidades internacionales, con base en la dosis y teniendo en cuenta la concentración y la presentación de los medicamentos.

c. Identificar el precio de la dosis y del tratamiento por persona, según el "caso base”.

Para establecer los precios según el sistema de información de precios de medicamentos (SISMED), se procedió de la siguiente manera.

1. Se buscó el correspondiente código único de medicamentos (CUM) para cada uno de los principios activos involucrados en el análisis, en las bases de datos de registros vigentes del listado del Instituto Nacional de Vigilancia de Medicamentos y Alimentos (Invima).

2. Los códigos obtenidos en el paso 1 se buscaron en la base de datos SISMED, estableciendo como periodo de extracción enero a junio de 2014.

3. Se utilizaron los siguientes filtros para los registros:

a. Tipo de precio: ventas (VEN)

b. Tipo de entidad: laboratorio (LAB). Solo en caso de no encontrar información después de filtrar por LAB, se incluyó el precio de mayoristas (MAY).

c. Canal: institucional (INS).

4. Después de usar este filtro, se tomó el promedio ponderado por el número de unidades reportadas, para las columnas "valor mínimo", "valor promedio” y "valor máximo".

5. El precio tomado para el "caso base" fue el promedio ponderado por el número de unidades reportadas.

Los medicamentos infliximab, adalimumab y etanercept, los cuales están sometidos a precio regulado por el Ministerio de Salud, se consultaron en la base de datos del SISMED de enero a junio 2014 y se verificó que el precio fuera similar al emitido en las circulares reguladoras de precios emitidas por el Ministerio de Salud ${ }^{13,14}$.

Los medicamentos se tasaron a precios del 2014. Para el metotrexato, se tomó el dato del 2013 y se actualizó, utilizando el índice de precios al consumidor (IPC) del 2014, reportado por el Departamento Administrativo Nacional de Estadística (DANE). 
Para determinar el costo por unidad mínima de consumo (tableta, cápsula, centímetro cúbico o ampollas), se tomó el valor de la presentación dividido por la cantidad. Para el caso de los medicamentos tópicos, se estimó la cantidad con base en la superficie corporal comprometida, de acuerdo con lo reportado en la historia clínica y con lo referenciado ${ }^{11}$.

Para los costos de medicamentos tópicos, sistémicos y productos biológicos, se asumió una duración de tratamiento de 24 semanas, con base en lo siguiente:

- En los ensayos clínicos, la eficacia de los medicamentos sistémicos y productos biológicos, habitualmente, se ha evaluado a las 24 semanas de tratamiento ${ }^{15,16}$.

- Los medicamentos tópicos se continúan a lo largo del tratamiento (en la fase de inducción y la de mantenimiento $)^{17}$.

- La fase de mantenimiento del tratamiento tópico está sujeta al criterio médico, según la evolución de la enfermedad, con base en la guía NICE CG153; este periodo comprende alrededor de 24 semanas $^{12}$.

- Las dosis utilizadas para el cálculo de los costos de los productos biológicos se basó en el esquema sugerido por la guía para psoriasis ${ }^{11}$, como sigue:

- $\quad$ Adalimumab CUM 19939766-1: por vía subcutánea, $80 \mathrm{mg}$ en la semana 0,40 mg en la semana 1 y, luego, $40 \mathrm{mg}$ cada 14 días.

- $\quad$ Etanercept CUM 19978841-7: por vía subcutánea, $50 \mathrm{mg}$ dos veces por semana hasta la semana $12 \mathrm{y}$, luego, $50 \mathrm{mg}$ cada semana.

- Infliximab CUM 19963298-1: $5 \mathrm{mg} / \mathrm{kg}$ intravenosos a las semanas o, 2 y 6, y luego, cada ocho semanas.

- Para calcular los costos de las consultas, los procedimientos y las ayudas diagnósticas, se tomaron las frecuencias de uso correspondiente a cada paciente multiplicada por el manual tarifario SOAT actualizado al año 2014, y el manual ISS 2001 más el $30 \%$, de acuerdo con la situación tarifaria. También, se utilizaron los promedios y las desviaciones estándar para hacer un análisis probabilístico de costos.

\section{Análisis de datos}

Se hizo un análisis probabilístico de los costos, utilizando los valores medios, mínimos, máximos y las desviaciones estándar de los costos de cada una de las actividades médicas. Mediante la programación Visual
Basic de Excel, se hicieron simulaciones de Montecarlo de mil iteraciones cada una, para cada uno de los componentes del costo de atención del paciente con psoriasis, incluyendo consultas médicas, medicamentos productos biológicos y no biológicos, ayudas diagnósticas y procedimientos. Con los resultados de la simulación de Montecarlo, se graficó la distribución probabilística de los costos totales. Esta última permite saber cuál es la probabilidad de que el tratamiento tenga un costo igual o inferior a una cifra determinada. En el análisis descriptivo se utilizaron frecuencias absolutas, relativas y medidas de resumen. El procesamiento y el análisis de los datos se hicieron mediante el software $\mathrm{SPSS}^{\circledR}$, versión 20, y Excel ${ }^{\circledR}$.

\section{RESULTADOS}

\section{Características sociodemográficas}

Se incluyeron 95 pacientes, de los cuales 47 eran mujeres; el rango de edad entre estuvo los 16 y los 80 años, con media de 47,6 años, desviación estándar (DE) de 15,3 , mediana de 48 y rango intercuartil de 26 años. La duración promedio de su tratamiento en la IPS fue de 688 días ( $D E=958)$, con mediana de 338 días y rango intercuartil de 659; el tiempo mínimo en el programa fue de 38 días y el máximo fue de 4.933 días. El 31,0 \% de los pacientes vivía en estratos 1 y 2, el 52,0 \% en estratos 3 y 4 y el 17,0 \% en estratos 5 y 6. De los 95, según el estado civil $48(50,5 \%)$ eran casados y, por condición de trabajo, 45 (47,4\%) eran empleados.

\section{Características clínicas}

El 53,6 \% (51) de los pacientes tenían afectación de su calidad de vida. El promedio del índice DLQI fue de 8,2 $(\mathrm{DE}=7,2)$ y el del PASI fue de 5,7 ( $\mathrm{DE}=4,7)$; de los 15 pacientes con PASI de 10 o más, todos tenían un DLQI de 10 o mayor. En los 9 pacientes en tratamiento biológico, el PASI promedio fue de $14(\mathrm{DE}=3,9)$ y el DLQI fue $\geq 10$. El 45,3 \% (43) de los pacientes tenía más de 10 años con la enfermedad y en 15,8 \% (15) la enfermedad fue grave según el PASI (TABLA 1). La forma clínica más común de presentación de la enfermedad fue la psoriasis en placas, $84,0 \%$, (76), seguida de la palmoplantar, 10,5\% (9), y la ungular, 6,0 \% (6), la pustulosa, 4,4 \% (4), y la inversa, 1 \% (1). Las enfermedades concomitantes más frecuentes fueron la hipertensión arterial sistémica (18,0 \%), la obesidad (16,1\%), el alcoholismo $(12,9 \%)$, la diabetes mellitus $(10,8 \%)$ y el tabaquismo $(8,6 \%)$. Todos los pacientes habían reci- 


\begin{tabular}{|c|c|c|c|}
\hline \multicolumn{2}{|c|}{ VARIABLE } & $\mathrm{n}$ & $\%$ \\
\hline \multirow{5}{*}{$\begin{array}{l}\text { Tiempo de evolución de } \\
\text { la enfermedad (años) }\end{array}$} & $>10$ & 43 & 45,3 \\
\hline & 7-9 & 15 & 15,8 \\
\hline & 4-6 & 17 & 17,9 \\
\hline & $1-3$ & 16 & 16,8 \\
\hline & $\ll 1$ & 4 & 4,2 \\
\hline \multirow{4}{*}{ BSA (\%) } & $>10$ & 25 & 26,3 \\
\hline & $6-9$ & 12 & 12,6 \\
\hline & $0-5$ & 51 & 53,7 \\
\hline & Datos perdidos & 7 & 7,4 \\
\hline \multirow{3}{*}{ PASI } & $\geq 10$ & 15 & 15,8 \\
\hline & $0-9$ & 71 & 74,7 \\
\hline & Datos perdidos & 9 & 9,5 \\
\hline \multirow{3}{*}{ DLQI } & $\geq 6$ & 37 & 38,9 \\
\hline & $\leq 5$ & 32 & 33,7 \\
\hline & Datos perdidos & 26 & 27,4 \\
\hline
\end{tabular}

BSA: body surface area; DLQI: Dermatology Life Quality Index; PASI: Psoriasis Area Severity Index
TABLA 1. Características clínicas de pacientes con psoriasis en CES, Sabaneta, 2014. $(\mathrm{N}=95)$.
TABLA 2. Tratamiento de 95 pacientes con psoriasis en CES, Sabaneta, $1^{\circ}$ de enero a 30 de noviembre de 2014

\begin{tabular}{cccc}
\hline TRATAMIENTO & & $n$ & $\%$ \\
Tópico & No & 29 & 30,5 \\
& Sí & 66 & 69,5 \\
Sistémico & No & 74 & 77,9 \\
Fototerapia & Sí & 21 & 22,1 \\
& No & 18 & 18,9 \\
Terapia biológica & Sí & 77 & 81,1 \\
& No & 86 & 90,5 \\
& Sí & 9 & 9,5 \\
\hline
\end{tabular}

bido tratamiento tópico antes del ingreso al programa. La distribución de los pacientes según el tratamiento se muestra en la (TABLA 2).

\section{Recursos utilizados}

La población de estudio utilizó 1.025 consultas ambulatorias durante 65.394 días-paciente. Las remisiones ambulatorias más frecuentes fueron a Reumatología (8,4 \%), a Psicología (8,4 \%), a Infectología (8,4\%) y a Nutrición (4,2 \%). Los exámenes de laboratorio más utilizados fueron el hemoleucograma, el perfil lipídico, las pruebas de función hepática y la creatinina. Los medicamentos tópicos más utilizados fueron el Daivobet ${ }^{\circledR}$
(52\%), la betametasona (34\%) y el clobetasol (26\%). En los tratamientos sistémicos, el metotrexato fue el más empleado (28\%), acompañado del ácido fólico como coadyuvante. De los productos biológicos, el más utilizado fue el etanercept (6\%) (TABLA 3).

\section{Costos del paciente con psoriasis}

El "caso base" equivale al tratamiento de un paciente con psoriasis leve o moderada a grave durante 24 semanas, con una fase inicial de tratamiento de inducción de 16 semanas y una de mantenimiento hasta la semana $24^{11,12}$. Se estableció la proporción de recursos consumidos en exámenes de laboratorio, consultas y 


\begin{tabular}{|c|c|c|}
\hline MEDICAMENTOS & n & $\begin{array}{c}\text { Probabilidad de } \\
\text { uso (\%) }\end{array}$ \\
\hline \multicolumn{3}{|l|}{ Tópicos } \\
\hline Daivobet $\circledast$ (calcipotriol + betametasona) & 49 & 0,52 \\
\hline Betametasona, crema & 32 & 0,34 \\
\hline Clobetasol & 25 & 0,26 \\
\hline Diprosalic $®$ (ácido salicílico + betametasona) & 8 & 0,08 \\
\hline Clobetasol + ácido salicílico + urea (magistral) & 8 & 0,08 \\
\hline Daivonex ${ }^{\circledR}$, crema (Calcipotriol) & 7 & 0,07 \\
\hline Hidrocortisona, crema & 5 & 0,05 \\
\hline Tazat $囚$ (tazaroteno) & 3 & 0,03 \\
\hline Desonisal ${ }^{(}$(desonida + ácido salicílico) & 2 & 0,02 \\
\hline \multicolumn{3}{|l|}{ Sistémicos } \\
\hline Metotrexato & 27 & 0,28 \\
\hline Acicretín & 5 & 0,05 \\
\hline \multicolumn{3}{|l|}{ Biológicos } \\
\hline Etanercept & 6 & 0,06 \\
\hline Adalimimab & 2 & 0,02 \\
\hline Infliximab & 1 & 0,01 \\
\hline \multicolumn{3}{|l|}{ Coadyuvantes } \\
\hline Ácido fólico & 27 & 0,28 \\
\hline
\end{tabular}

TABLA 3. Probabilidad de uso de medicamentos en pacientes con psoriasis, en CES, Sabaneta, 2001$2014(\mathrm{~N}=95)$.

\begin{tabular}{ccccc}
\hline & \multicolumn{2}{c}{ Manual ISS 2001 $+30 \%$} & \multicolumn{2}{c}{ Manual SOAT } \\
TIPO DE PSORIASIS & Mínimo & Máximo & Mínimo & Máximo \\
Leve & $\$ 21.710$ & $\$ 13^{\prime} 803.505$ & $\$ 35.500$ & $\$ 16^{\prime} 493.678$ \\
Moderada a grave & $\$ 402.610$ & $\$ 36^{\prime} 643.757$ & $\$ 536.987$ & $\$ 39^{\prime} 312.273$ \\
En tratamiento no biológico & $\$ 21.710$ & $\$ 17^{\prime} 290.244$ & $\$ 35.500$ & $\$ 24^{\prime} 050.138$ \\
En terapia biológica & $\$ 15^{\prime} 608.272$ & $\$ 36^{\prime} 643.757$ & $\$ 17.286 .259$ & $\$ 39^{\prime} 312.272$ \\
\hline
\end{tabular}

TABLA 4. Costo en COP de medicamentos no biológicos (tópicos y sistémicos), fototerapia, productos biológicos (etanercept, adalimumab e infliximab), consultas médica y exámenes de laboratorio, de los pacientes con psoriasis atendidos en CES, Sabaneta, durante 24 semanas.

procedimientos durante las 24 semanas (168 días), teniendo como tiempo de referencia el promedio en el programa (688 días).

El costo de 24 semanas de tratamiento de un paciente con psoriasis, según el manual ISS, varió de un mínimo de COP\$ 16.263 (equivalente a una consulta) a un máximo de COP\$ 4'614.682; según el manual SOAT, el costo estuvo entre COP\$ 35.500 y COP\$ 5'682.864. Al analizar los costos durante las 24 semanas, en el subgrupo de pacientes que recibieron terapia biológica, se calculó un costo ISS mínimo de COP\$15'608.279 y un máximo de COP\$ 36'643.757; según el manual SOAT, estuvieron entre COP\$ 17'296.259 y COP\$ 39'312.273 (TABLA 4). Al analizar el costo para pacientes que no recibieron tratamiento biológico, se encontraron, según el manual ISS, un mínimo de COP\$ 16.263 y un máximo de COP\$ 17'290.244; según el manual SOAT, el costo mínimo fue de COP\$ 35.500 y el máximo fue de COP\$24’050.138. 


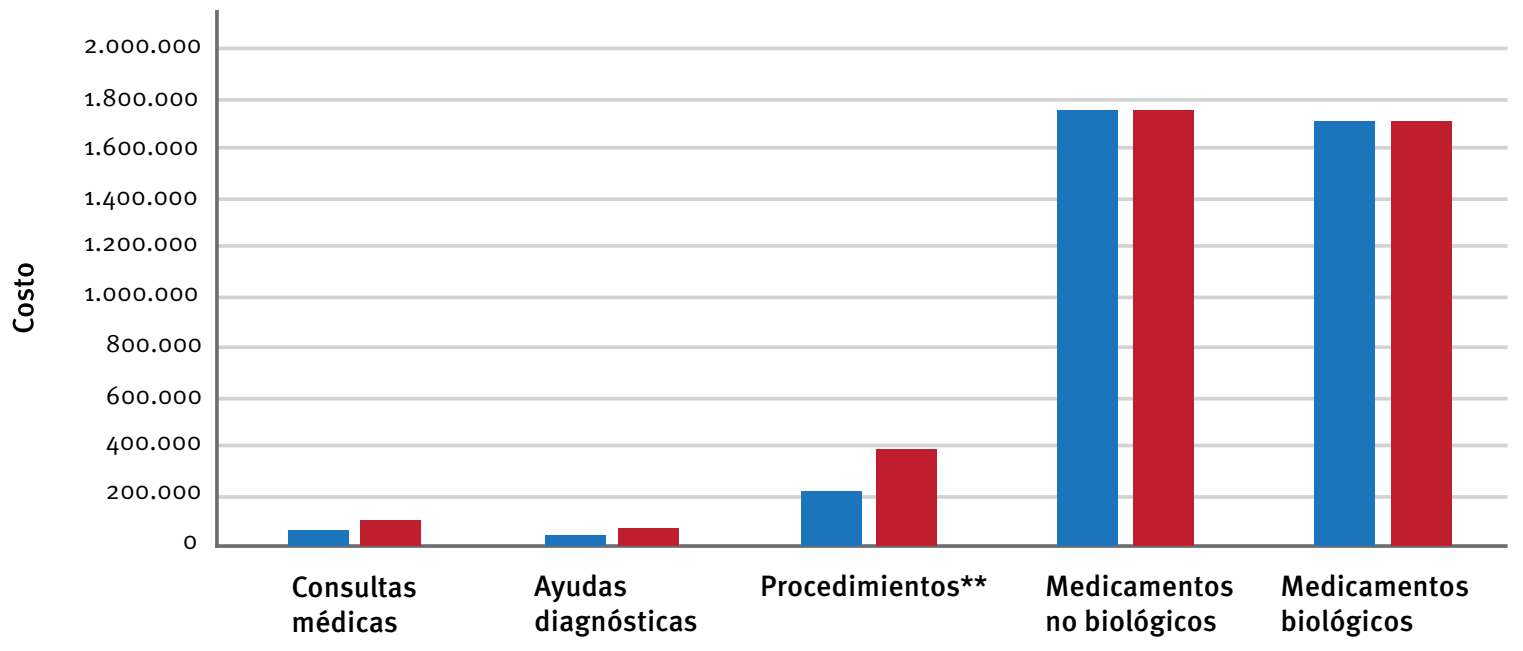

ISS $+30 \% \quad$ SOAT

FIGURA 1. Distribución del costo total de tratamiento de pacientes con psoriasis durante 24 semanas.

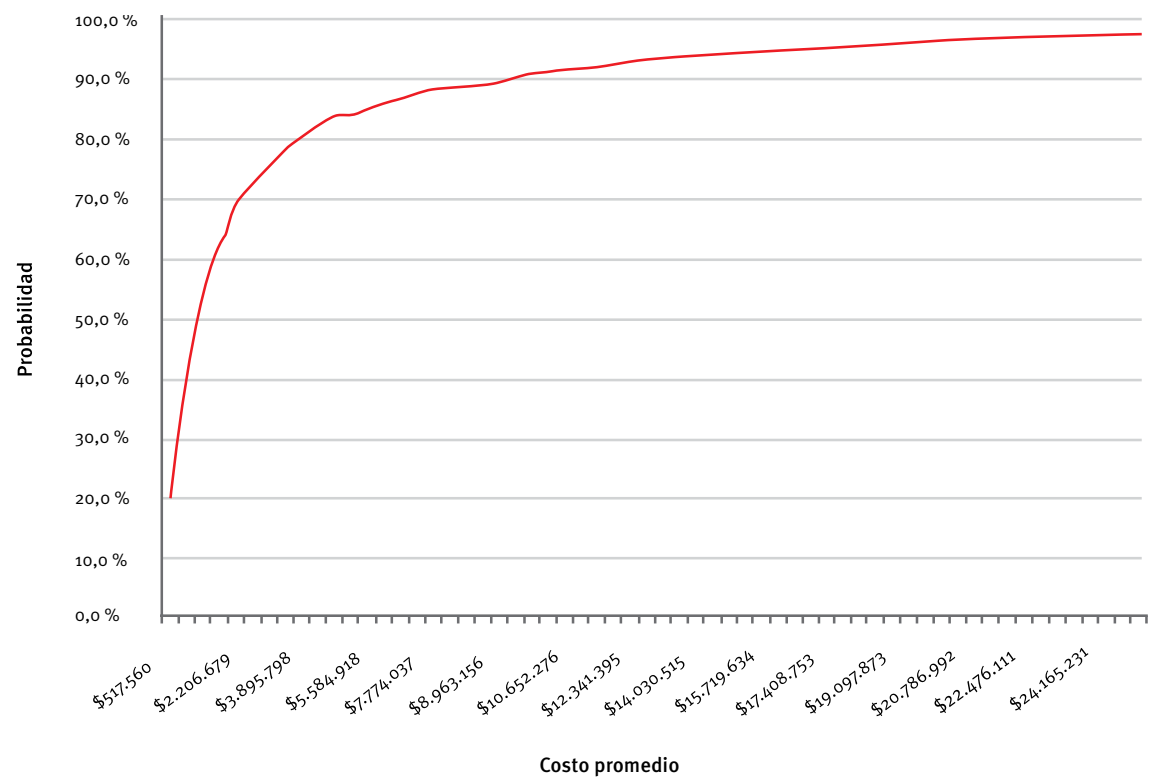

FigURA 2. Distribución probabilística de costos de un caso base durante 24 semanas de tratamiento.

Los costos de los pacientes con psoriasis, según el manual ISS y el SOAT, fueron mayores en este último, especialmente por los ítems de consultas médicas ambulatorias, ayudas diagnósticas y procedimientos (fototerapia) (FIGURA 1).

La distribución probabilística acumulada permite observar la probabilidad de que un determinado costo total promedio sea igual o inferior a un valor dado. La probabilidad de que el costo de la atención de un paciente sea de COP\$10'229.996 (cruzado por la línea punteada) o menor, es de $90 \%$. De igual forma, se puede estimar para cualquier otro valor (FIGURA 2).

La distribución probabilística acumulada permite observar la probabilidad de que un determinado costo total promedio sea igual o inferior a un valor dado. La probabilidad de que el costo de atención de un paciente sea de COP\$10'229.996 (cruzado por la línea punteada) o menor, es de $90 \%$. 


\section{DISCUSIÓN}

En este estudio se abordó la psoriasis de manera general, con el fin de hacer una caracterización macroscópica del perfil de las personas afectadas por la psoriasis en un centro de referencia del área metropolitana del Valle de Aburrá, que pueda servir de referente para el resto del país.

Los estudios en Colombia revelan que la prevalencia de la psoriasis es mayor en el grupo de edad entre los 43,6 y los 54,5 años, en personas casadas, y en la población mestiza y blanca. La duración de la enfermedad es de 14 años, con una media de 9,5, y un PASI entre 7 y 12. La principal enfermedad concomitante es la hipertensión arterial, y son frecuentes el tabaquismo y el consumo de alcohol ${ }^{18,19}$.

Las enfermedades concomitantes son comunes en los pacientes con psoriasis. Los hallazgos del presente estudio concuerdan con los reportes de alta prevalencia de obesidad ${ }^{20}$, hipertensión arterial sistémica ${ }^{21-25}$, diabetes $^{26,27}$ y dislipidemia ${ }^{28}$.

Al momento del estudio, el 68,0 \% de los pacientes recibía tratamiento tópico; es de aclarar que no se dispone de un registro que indique el motivo por el cual otros pacientes no lo recibían. Sin embargo, es de suponer que muchos pacientes habían recibido periodos muy largos de tratamiento tópico antes de su ingreso a la IPS, lo que pudo llevar a taquifilaxia o a efectos secundarios, obligando al médico a suspender el tratamiento por un tiempo razonable; además, su eficacia y el poco cumplimiento del tratamiento, hace que los pacientes lo abandonen.

La afectación de la calidad de vida es común en casos de psoriasis; hasta el 79,0 \% de los pacientes manifiestan un impacto negativo en su vida por la enfermedad $^{29}$. En el presente estudio, se observó que el $38,9 \%$ (37) de los pacientes tenía algún grado de afectación en su calidad de vida (DLQI>5), con un DLQI promedio de 8,2; en los estudios previos de Vélez, et al., el DLQI promedio fue de 3,2 (IC $95 \%: 2,4-3,9)^{18}$. Esto puede obedecer a que el 45,3 \% de los pacientes con psoriasis llevan 10 años o más de evolución de la enfermedad y, a pesar de que la mayoría de las formas clínicas son leves, la alteración de su calidad de vida es considerable, Además, como la IPS es un centro de referencia especializado en esta enfermedad, muchos pacientes han requeridos múltiples tratamientos con grados variables de efectividad. De ahí la importancia de tratar a estos pacientes de manera multidisciplinaria y en forma conjunta con el servicio de Psicología ${ }^{11}$.

Las formas clínicas de presentación de la enfermedad son coherentes con los estudios previos desarrollados en Colombia ${ }^{18,19,30}$. El número de pacientes con terapia biológica es bajo, a pesar de ser un centro donde se maneja un volumen alto. Solo alrededor del 9,5\% de ellos están incluidos en esta modalidad terapéutica, lo cual se relaciona exactamente con la gravedad de la enfermedad (leve), considerando, además, que el ingreso de estos pacientes a los tratamientos con productos biológicos se decide de manera conjunta con el equipo de dermatólogos de la IPS y no según un criterio individual, evitando usarlos cuando no están indicados. Otra de las razones para no usarlos es la buena respuesta terapéutica obtenida con la fototerapia; la terapia biológica solamente se administra a los pacientes que no mejoran con la radiación UV.

\section{Costos}

Los estudios de costos de psoriasis se centran principalmente en la evaluación de costo-efectividad de los tratamientos con productos biológicos, en enfermedad moderada a grave, por lo cual, son escasos los estudios de costos desarrollados bajo la práctica habitual. Los resultados en pacientes ambulatorios con psoriasis no son comparables entre sí, porque los análisis se hacen desde diferentes perspectivas, las fuentes de información son diferentes, el sistema de salud es distinto y no se tienen en cuenta variables como la extensión del área corporal; además, algunos pacientes no cumplen con el tratamiento y su eficacia es variable.

$\mathrm{Sohn}^{8}$, en un estudio retrospectivo del costo de la psoriasis moderada a grave en Alemania, desde una perspectiva social (incluidos todos los costos médicos, no médicos, directos e indirectos sin importar quién paga por el recurso), encontró que el costo promedio ascendió a € 6.709 por paciente por año. En general, la media anual de costos de atención ambulatoria fueron de $€ 204$ (DE=€ 188), para el subgrupo de pacientes sin tratamiento sistémico, de €224 (DE=€ 199), para el subgrupo con tratamiento sistémico, y de $€ 199$ ( $D E=139$ ), para el subgrupo de pacientes de alta necesidad (highneed patients). Los principales costos fueron los de la consulta, los de la fototerapia y los de las pruebas de laboratorio.

Colombo ${ }^{31}$, en Italia, en un estudio nacional, multicéntrico y prospectivo, de tres meses de evaluación de los costos de psoriasis en pacientes con estado moderado y grave de la enfermedad (desde la perspectiva del paciente, de un tercer pagador (National Health Service, NHS, y de la sociedad), encontró que el costo medio total para la psoriasis, incluidos los costos directos e indirectos, era de $€ 8.371,61$ por paciente por año. El costo medio para aquellos con enfermedad moderada 


\section{"Los estudios de costos de psoriasis se centran principalmente en la evaluación de costo-efectividad de los tratamientos con productos biológicos, en enfermedad moderada a grave, por lo cual, son escasos los estudios de costos desarrollados bajo la práctica habitual".}

(PASI $\leq 20$ ) fue de $€$ 5.226,04, mientras que, para los pacientes con enfermedad más grave (PASI>20) fue de $€$ $11.434,40$ al año.

Vanderpuye-Orgle, et al.32, en una revisión sistemática sobre la carga económica de la psoriasis en Estados Unidos, determinaron que el costo anual de la enfermedad era de USD \$ 35,2 billones de dólares, considerando una población afectada por la psoriasis de 7,4 millones de personas en 2013. Los costos indirectos generados por la enfermedad y atribuidos a la alteración en el DLQI, era de USD\$11,8 billones y los costos atribuidos a pérdida laboral fueron de USD \$11,2 billones.

Los tratamientos con productos biológicos han revolucionado el manejo de la psoriasis en el mundo y, por lo tanto, han aumentado los costos, ya que estos medicamentos son de alto valor, aunque reducen los días de hospitalización, el uso de otros tratamientos sistémicos, mejoran el PASI considerablemente y aumentan la satisfacción del paciente. En un estudio realizado por Cheng, et al. ${ }^{33}$ sobre el costo de los productos biológicos para la psoriasis, demostró que los valores se incrementan durante el primer año, ya que las dosis de impregnación son mayores; son de USD\$ 46.395 contra USD\$ 37.111 para el etanercept, de USD\$ 36.038 para el adalimumab y de USD\$ 53.909 frente a USD\$ 44.925 para el ustekinumab. Entre 2004 y 2014, el costo de estos medicamentos aumentó $120 \%$ para el etanercept, $103 \%$ para el adalimumab y $53 \%$ para el ustekinumab.

Los costos totales del paciente con psoriasis moderada a grave en este estudio probablemente no reflejen la realidad, por los siguientes aspectos:

Los pacientes a los que se les formula un medica- mento biológico, siguen un control específico en su EPS con un médico a cargo de hacerle seguimiento al tratamiento; por lo tanto, los recursos utilizados y los costos derivados de este seguimiento son desconocidos para el Centro Dermatológico CES, Sabaneta.

Los pacientes son controlados para las enfermedades asociadas en su EPS y los exámenes de laboratorio derivados del control también son desconocidos para el Centro Dermatológico CES, Sabaneta.

La fototerapia y el tratamiento combinado con esteroides son un factor importante en la reducción del costo. La fototerapia puede incrementar en $20 \%$ el costo, pero no se equipara con los costos de la terapia biológica ${ }^{34}$.

En una revisión sistemática de D’Souza, et al., se encontró que de los tratamientos sistémicos para la psoriasis, incluyendo los medicamentos convencionales no biológicos y los biológicos, el metotrexato era el de menor costo, seguido de la ciclosporina, la fototerapia con UVB y el acitretín, y un mayor costo de la fototerapia con PUVA. Entre los productos biológicos estaban en, primer lugar, el ustekinumab a una dosis de $90 \mathrm{mg}$, seguido del infliximab, el etanercept y el ustekinumab a una dosis de $45 \mathrm{mg}$, y finalmente, el adalimumab, a una dosis de $40 \mathrm{mg}^{35}$.

Galadari ${ }^{10}$, con datos de 1994 , encontró que el costo medio de tratamiento de la psoriasis era de $€ 905$. Jener ${ }^{6}$, en Australia, analizó 83 pacientes con psoriasis, seguidos durante un período de dos años; el gasto anual de bolsillo para los productos médicos fue de alrededor de AUD\$250 por persona, con costos que iban de cero a más de AUD\$2.00o por persona du- 
rante el período de dos años. Los valores fueron más altos para los productos adquiridos sin receta médica (over-the-counter). Javitz ${ }^{7}$ estimó el costo directo de la atención médica para la psoriasis (incluyendo la artritis psoriásica) desde una perspectiva social, entre los adultos de los Estados Unidos. El costo aproximado de la enfermedad clínicamente significativa en 1,4 millones de personas es sustancial: USD\$30,5 millones en hospitalizaciones, USD\$ 86'600.00o en visitas ambulatorias, USD\$27'400.00o en fotoquimioterapia, USD\$ 147 '900.000 en los medicamentos recetados y USD\$ 357 '200.000 para los de venta libre, para un total de USD\$ 649,6 millones.

Los resultados de los costos en el presente estudio, si bien no se pueden comparar con los estudios disponibles, están dentro de lo esperado según la bibliografía consultada; además, los resultados indican que la psoriasis tiene considerables consecuencias económicas para el sistema de salud.

La limitación de este estudio se debe a que no se hicieron ajustes según el incumplimiento del tratamiento, lo cual, obviamente, disminuye su efectividad; además, el desconocimiento de los costos derivados del tratamiento biológico pudo llevar a subestimar los valores.

Las fortalezas de este estudio son que la información proviene de un centro de referencia en el manejo de la enfermedad, que los datos son de la práctica habitual (real life data), que se creó un "caso base” según las guías de referencia para el tratamiento de la enfermedad $^{11,12}$, y que el ajuste del tratamiento según la efectividad y el área corporal comprometida da mayor validez a los resultados.

\section{CONCLUSIONES}

La psoriasis es una enfermedad crónica que afecta la calidad de vida del paciente, con unos costos de atención importantes para el sistema de salud, especialmente por los productos biológicos, por lo que es importante utilizar tratamientos efectivos para una rápida remisión de la enfermedad y evitar la presencia de enfermedades concomitantes.

Los principales costos corresponden a las formas moderadas a graves de la psoriasis y se relacionan con los tratamientos sistémicos convencionales o con productos biológicos

El costo de los tratamientos biológicos debe analizarse desde el punto de vista de su costo-efectividad, lo cual debe ser considerarse en las guías de tratamiento elaboradas en cada uno de los países.

\section{Agradecimientos}

Al Grupo CES Dermatología, a la Facultad de Psicología de la Universidad CES y al Laboratorio Novartis de Colombia, S.A.

\section{REFERENCIAS}

1. Christophers E. Psoriasis--epidemiology and clinical spectrum. Clin Exp Dermatol. 2001;26:314-20.

2. Lowes MA, Bowcock AM, Krueger JG. Pathogenesis and therapy of psoriasis. Nature. 2007; 445:866-73.

3. Krueger JG. The immunologic basis for the treatment of psoriasis with new biologic agents. J Am Acad Dermatol. 2002;46:1-26.

4. Franco-Aguirre JQ, Cardona-Arias JA. Characterization of health-related quality of life studies in people with psoriasis: Systematic review 2003-2013. Rev Colomb Reumatol. 2014;21:35-41.

5. Ordoñez J, Palacios A, Londoño A, Jiménez S. Medición de la calidad de vida por medio del Dermatology Life Quality Index en pacientes con psoriasis: una revisión sistemática. Rev Asoc Colomb Dermatol. 2013;21:305-13.

6. Jenner N, Campbell J, Plunkett A, Marks R. Cost of psoriasis: a study on the morbidity and financial effects of having psoriasis in Australia. Australas J Dermatol. 2002;43:255-61.

7. Javitz HS, Ward MM, Farber E, Nail L, Vallow SG. The direct cost of care for psoriasis and psoriatic arthritis in the United States. J Am Acad Dermatol. 2002;46:850-60.

8. Sohn S, Schoeffski O, Prinz J, Reich K, Schubert E, Waldorf K, et al. Cost of moderate to severe plaque psoriasis in Germany: A multicenter cost-of-illness study. Dermatol Basel Switz. 2006;212:137-44.

9. Finzi AF, Mantovani LG, Belisari A, Italian Association for Studies on Psoriasis. The cost of hospital-related care of patients with psoriasis in Italy based on the AISP study. J Eur Acad Dermatol Venereol JEADV. 2001;15:320-4.

10. Galadari I, Rigel E, Lebwohl M. The cost of psoriasis treatment. J Eur Acad Dermatol Venereol JEADV. 2001;15:290-1.

11. Asociación Colombiana de Dermatología y Cirugía Dermatológica, Grupo Colombiano de Psoriasis y Artritis Psoriásica. Guías Manejo de la Psoriasis en Colombia. Fecha de consulta: 2 de diciembre de 2014. Disponible en: http://www.solapso. org/archivos/guia.pdf.

12. National Institute for Health and Care Excellence (NICE). Psoriasis: Assessment and management of psoriasis (CG 153). Fecha de consulta: 21 de enero de 2015. Disponible en: https://www.nice.org.uk/guidance/cg153.

13. Ministerio de Salud y Protección Social, Comisión Nacional de Medicamentos y Dispositivos Médicos. Circular o4 de 2013. Fecha de consulta: 21 de enero de 2015. Disponible en: http://www.achc.org.co/documentos/afiliados/Circulares/ Circular\%2004\%2ode\%202013\%20CNPMDM.pdf.

14. Ministerio de Salud y Protección Social. Regulación de precios de medicamentos. Fecha de consulta: 21 de enero de 2015. Disponible en: https://www.minsalud.gov.co/salud/ MT/Paginas/medicamentos-regulacion-precios.aspx. 
15. Lucka TC, Pathirana D, Sammain A, Bachmann F, Rosumeck S, Erdmann R, et al. Efficacy of systemic therapies for moderate-to-severe psoriasis: a systematic review and meta-analysis of long-term treatment. J Eur Acad Dermatol Venereol JEADV. 2012;26:1331-44.

16. Schmitt J, Rosumeck S, Thomaschewski G, Sporbeck B, Haufe E, Nast A. Efficacy and safety of systemic treatments for moderate-to-severe psoriasis: Meta-analysis of randomized controlled trials. Br J Dermatol. 2014;170:274-303.

17. Mason J, Mason A, Cork M. Topical preparations for the treatment of psoriasis: A systematic review. Fecha de consulta: 21 de enero de 2015. Disponible en: https://www.york. ac.uk/che/pdf/op41.pdf.

18. Vélez N, Bohórquez L, Corrales MF, Orrego S, Jiménez SB, Zuluaga de Cadena Á, et al. Caracterización de los pacientes con psoriasis en tratamiento con fototerapia. Rev Asoc Colomb Dermatol. 2011;19:100-6.

19. González C, Castro L, De La Cruz G, Arenas CM, Beltrán A, Santos AM. Caracterización epidemiológica de la psoriasis en el Hospital Militar Central. Rev Asoc Col Dermatol. 2009;17:11-7.

20. Carrascosa JM, Rocamora V, Fernandez-Torres RM, JimenezPuya R, Moreno JC, Coll-Puigserver N, et al. Obesity and psoriasis: inflammatory nature of obesity, relationship between psoriasis and obesity, and therapeutic implications. Actas Dermo-Sifiliográficas. 2014;105:31-44.

21. Armstrong AW, Harskamp CT, Armstrong EJ. The association between psoriasis and hypertension: A systematic review and meta-analysis of observational studies. J Hypertens. 2013;31:433-43.

22. Carbo Amoroso E, Leban V, Federico D, Williner ME, Díaz MG, Guardati MV, et al. Psoriasis y síndrome metabólico: Estudio retrospectivo sobre 22 casos. Rev Argent Dermatol. 2010;91. Fecha de consulta: 2 de diciembre de 2014. Disponible en: http://www.scielo.org.ar/scielo.php?script=sci arttext\&pid=S1851-300X2010000100002.

23. Huski J, Alendar F. Tissue angiotensin-converting enzyme in patients with various clinical forms of psoriasis. Bosn J Basic Med Sci Udruženje BasiØnih Med Znan Assoc Basic Med Sci. 2007;7:103-6.

24. Liu T, Han Y, Lu L. Angiotensin-converting enzyme gene polymorphisms and the risk of psoriasis: a meta-analysis. Clin Exp Dermatol. 2013;38:352-59.

25. Ryder KW, Epinette WW, Jay SJ, Ransburg RC, Glick MR. Serum angiotensin converting enzyme activity in patients with psoriasis. Clin Chim Acta Int J Clin Chem. 1985;153:143-6.

26. Brauchli YB, Jick SS, Meier CR. Psoriasis and the risk of incident diabetes mellitus: A population-based study. $\mathrm{Br} \mathrm{J}$ Dermatol. 2008;159:1331-7.

27. Wellen KE, Hotamisligil GS. Inflammation, stress, and diabetes. J Clin Invest. 2005;115:1111-9.

28. Ma C, Harskamp CT, Armstrong EJ, Armstrong AW. The association between psoriasis and dyslipidaemia: a systematic review. Br J Dermatol. 2013;168:486-95.

29. de Arruda LH, De Moraes AP. The impact of psoriasis on quality of life. Br J Dermatol. 2001;144(Suppl.58):33-6.

30. Valencia C, Vélez H, Santamaría L, Vargas M, Montoya F. Psoriasis y dermatomicosis. Iatreia. 1994;7:18-20.

31. Colombo G, Altomare G, Peris K, Martini P, Quarta G, Congedo $\mathrm{M}$, et al. Moderate and severe plaque psoriasis: Cost-of-illness study in Italy. Ther Clin Risk Manag. 2008;4:559-68.
32. Vanderpuye-Orgle J, Zhao Y, Lu J, Shrestha A, Sexton A, Seabury S, et al. Evaluating the economic burden of psoriasis in the United States. J Am Acad Dermatol. 2015;72:961-7.

33. Cheng J, Feldman S. The cost of biologics for psoriasis is increasing. Drugs Context. 2014;3:1-10.

34. Mustonen A, Leino M, Mattila K, Koulu L, Tuominen R. Treatment costs of psoriasis in a tertiary-level clinic. BMC Health Serv Res. 2014;14:344.

35. D'Souza LS, Payette MJ. Estimated cost efficacy of systemic treatments that are approved by the US Food and Drug Administration for the treatment of moderate to severe psoriasis. J Am Acad Dermatol. 2015;72:589-98. 